Rebekka Hufendiek

\title{
William James and John Dewey on Embodied Action-Oriented Emotions
}

\begin{abstract}
In this paper I present William James's theory of emotions and the central role it ascribes to the bodily reactions in emotions. I call the claim that there is a distinct pattern of bodily reactions that is constitutive for an emotion type the essential-ingredient view. I discuss some of the famous objections that cognitivists have raised against the feeling theory and characterize the position that cognitivist accounts take with regard to bodily reactions in emotions as the coincidental-byproduct view. With regard to current empirical research from psychophysiology I argue that the coincidental-byproduct view and the essential-ingredient view are both untenable. Yet the Jamesian view can be understood in a broader context as not aiming to find essential ingredients of emotions but rather taking the bodily arousal to be part of an organisms preparing for action. A reinterpretation of James in this manner has already been presented by John Dewey, who also provides a broader framework to think of emotion and perception as being co-constituted by action-tendencies.
\end{abstract}

\section{James and the essential-ingredient view}

In his classic essay What is an Emotion? (1884), William James argues that the feeling of bodily arousal is what constitutes an emotion. The commonsense view says that emotions simply cause bodily arousal; when we are sad, we therefore start crying or when we feel embarrassed, we blush. Yet James inverts the commonsense view and argues that in most cases we perceive something, like a dangerous predator or an offensive opponent, and this perception directly triggers bodily arousal, which is felt and evaluated only afterwards. James's central argument for this is of a phenomenological character: if you imagine a strong emotion and try to subtract the feeling of all involved bodily symptoms, nothing remains "but a cold and neutral mental state of intellectual perception" (James 1884, 193). We can refer to this argument as the "subtraction argument".

Upon a closer look, however, James makes two related claims. The first claim is that a mental state that does not involve the perception of bodily arousal would not be classified as an emotion at all. The second claim is that different kinds of emotions owe their special character to the different kinds of bodily arousal that constitute them. Both claims are nicely illustrated in the following 
quote: "What kind of an emotion of fear would be left, if the feelings neither of quickened heart-beats nor of shallow breathing, neither of trembling lips nor of weakened limbs, neither of goose-flesh nor of visceral stirrings, were present, it is impossible to think" (James 1884, 193f.). These are some of the physiological elements involved in fear. Rage according to James is unimaginable with "no ebullition of it in the chest, no flushing of the face, no dilatation of the nostrils, no clenching of the teeth, no impulse vigorous to action, but in their stead limp muscles, calm breathing, and a placid face" (James 1884, 194). The bodily processes involved in emotions are their essential ingredients. Subtract them away and the mental state that remains is classifiable neither as a state of fear nor as an emotion at all. We can refer to this as the "essential-ingredient view." James's essential-ingredient view has been the target of many objections, which I discuss below. ${ }^{1}$

\section{Nussbaum and the coincidental-byproduct view}

Cognitivist approaches have criticized James view not only for ignoring the cognitive dimension of emotions but also for overestimating the role of bodily arousal in emotional episodes. Nussbaum (2001) argues that while emotions involve bodily sensations of many kinds, there are no constant correlations between specific bodily processes and certain kinds of feelings, thus bodily reactions appear to be a coincidental byproduct of emotions and not an essential ingredient. We can refer to this view as the "coincidental-byproduct view." Nussbaum's position represents a broad consensus among cognitivists. While cognitivists tend to argue that emotions are constituted or caused by cognitive states such as beliefs or judgments, they tend to downplay the role of the body in emotional processing. ${ }^{2}$ With this view cognitivists such as Nussbaum stand in the tradition of the majority of authors in the second half of the 20th century in philosophy of mind and cognitive science, who take cognition to be disembodied.

1 Also, James' theory of emotions has been interpreted in various ways, some of which are perhaps more charitable than the present one. In this paper I focus on a systematic question concerning the standard reading of James' feeling theory. But see e.g. Ellsworth (2014) for the claim that James never aimed to present distinct emotion categories or Ratcliffe (2005) for the claim that James can account for the intentionality of emotions.

2 In this spirit Robert Solomon (with regard to his early works from the 70's) remarks, "In my original theory, it was by no means clear, that the body had any essential role in emotions" (Solomon 2003, 189). 
Nussbaum puts forward a conceptual and an empirical argument in defense of the coincidental byproduct view. The conceptual argument, as Nussbaum articulates it, employs the possibility of bodiless creatures having emotions: "Whether we believe that bodiless substances exist or not, the reason it makes sense to imagine a bodiless substance having genuine emotions is that it makes sense to imagine that a thinking being, whether realized in matter or not, could care deeply about something in the world, and have thoughts and intentions associated with such attachments. And that's all we really require for emotion" (Nussbaum 2001, 60). Nussbaum argues here that it is conceptually possible to think of an emotion as an intense state of mind that is not realized in any kind of matter at all. Actually, James happily admits that it is no "contradiction in the nature of things, or that pure spirits are necessarily condemned to cold intellectual lives" (James 1884, 194). So, with regard to this particular argument there is no real disagreement between the coincidental byproduct and the essential ingredient view. What James instead wants to claim is that "for us, emotion dissociated from all bodily feeling is inconceivable" (James 1884, 194, emphasis added). After all, from a naturalist point of view, the mere conceptual possibility of disembodied emotions in possible worlds where thinking substances without a body exist, can be conceded. But what is of interest is how emotions are realized in living organisms in the actual world. And here, the hypothesis at stake is that to "care deeply" about something or someone involves specific bodily processing such as the release of oxytocin.

Therefore, it is the empirical argument that really is of importance for the evaluation of the Jamesian account. Walter Cannon (1927) did research to test the Jamesian hypothesis as early as the 1920 s. In his experiments, the spinal cord and vagus nerve in dogs and cats were cut to inhibit feedback from the body to the brain. The animals were tested with regard to their reactions to emotional stimuli like threatening shocks. Their ability to react emotionally to such stimuli appeared to be intact. According to Cannon, the animal tests prove that even if you cut the bodily feedback entirely, feelings do not seem to be reduced. Even today, Cannon's article ranks high on the list of the most quoted evidence for all variations of the coincidental-byproduct view. ${ }^{3}$ It is often combined in one vein with a study by Chwalisz, Diener, and Gallagher (1988) who tested patients with spinal cord injuries. The outcome suggests that their emotional lives remain relatively stable. However, as William James already knew, cutting the vagus nerve and the spinal cord alone is not enough to prove him wrong:

3 See for example Solomon 2001/2003, 26. 
A crucial test of the truth of the hypothesis is quite as hard to obtain as its decisive refutation. A case of complete internal and external corporeal anaesthesia, without motor-alteration or alteration of intelligence except emotional apathy would afford, if not a crucial test, at least a strong presumption, in favour of the truth of the view we have set forth; whilst the persistence of strong emotional feeling in such a case would completely overthrow our case (James 1884, 203).

More recently, Antonio Damasio (1999) carefully detailed the paths bodily feedback can take and which forms of damage could eliminate or reduce it. Damasio points out that an embodied account can include more than just the bodily reactions in the visceral organs. Musculoskeletal feedback from the body and the face is apparently quicker and contributes to interoception during emotions. That spinal cord injury patients do not lose the ability to feel emotions can be explained by the fact that in these patients the vagus nerve usually is still transporting information. Also, only a fraction of the enactment of emotions depends on the spinal cord. A large number of processes are mediated by cranial nerves at the brain-stem level that can act on the face and on the viscera, and by other brain-stem nuclei that can act directly on the parts of the brain above their own level. Also, a significant part of bodily feedback does not travel via the nerves at all, but by way of the bloodstream from where it directly enters the brainstem. Nevertheless, that the reduced amount of bodily feedback might also reduce emotional feelings is suggested by Hohmann (1966), who interviewed patients with a spinal cord lesion and found that they reported subdued experience for many emotions except sadness, and also found a correlation between the location of the spinal lesion and the degree to which their emotional experience was reduced. But even if the location of the lesion is high on the spine, this does not suggest that patients should entirely lack emotional feelings, since the body does not end above the neck. Face, skull, oral cavity, and tongue provide a massive input into the brain that enters at brain-stem level and cannot be inhibited by any spinal cord injury. Furthermore, most emotions express themselves in changes of the facial musculature, the musculature of the throat, and in autonomic changes of the skin in the face and scalp. The representation of these changes in the brain remains available even with spinal cord injuries (Damasio 1999, 290).

Similar arguments can be brought up against Cannon's interpretation of the animal experiments. Why should Cannon have expected his prediction, that cats or dogs with severed spinal cord and vagus nerve should have a complete loss of emotional display, to be true in the first place? A severed vagus nerve and spinal cord do not impede the pathways for the facial responses that come from the brain-stem, and are mediated by cranial nerves which were not compromised in Sherrington's or Cannon's experiments. It's therefore not surprising that the 
cats and dogs in the study showed anger expressions, even if they could not move their bodies (Damasio 1999, 291).

Another anti-Jamesian argument is that bodily reactions cannot be constitutive of emotions, since the reduction of bodily feedback does not always lead to reduced emotional experience. Yet the studies cited to prove this claim are, at the very least, highly ambivalent. Reisenzein and Stephan $(2014,43)$ argue, for example, that studies in which facial feedback has been partly blocked by Botox injections (Davis, Sengas, Brandt, Ochsner 2010) do not support the claim that facial feedback is necessary for emotions. Yet this is not what James or typical current views claim. The claim is that emotions (and thereby emotional feelings) are constituted through patterns of bodily arousal. The absence of one component of the bodily reaction should have an effect on the realization of the emotion and might also effect how the emotion feels but should not necessarily prevent its occurrence. This theoretical prediction fits with the results of the studies quite well. They show that subjects who received a Botox injection showed emotional muting in response to a mildly amusing video clip, while no such response was observed in strongly negative or positive clips. A plausible interpretation of this result is that in the case of strongly emotion-eliciting clips there is no remarkable difference in experience because there are many bodily reactions going on, while in the case of mild joy the feedback that stems from a smile is a central factor of the bodily reaction so that its absence makes a difference. Similar arguments can be made for other cases of reduced bodily feedback as well. ${ }^{4}$

With regard to the second claim, that particular kinds of emotions owe their special character to the pattern of bodily arousal that constitutes them, Nussbaum presents phenomenological and empirical arguments in favor of the coincidental-byproduct view. While, as we have seen, James considers the reactions of the nervous system in fear and rage to be distinct, according to Nussbaum the feeling of bodily arousal involved in an emotion can vary. While "many men report experiencing anger in connection with a boiling feeling," Nussbaum states, "my own experience of anger is that it is associated with tension at the back of the neck, or a headache that appears the next day" (Nussbaum 2001, 61). This phenomenological self-observation is meant to refute the idea that every type of emotion is associated with a specific kind of bodily arousal. Whether rage is associated with boiling blood, or tensed muscles around the neck, or another bodily reaction, or no bodily reaction at all can vary from person to person and is therefore no criterion by which individuate emotions. From merely the phenomenological point of view the disagreement between the essential-ingredient view

4 See Laird and Lacasse 2014 for further arguments. 
and the coincidental-byproduct view seems hard to decide, as reports in favor of either view can be found.

Nussbaum's argument appears to find support in the studies done by Stanley Schachter and Jerome Singer (1962), who injected subjects with adrenaline and exposed them to different scenarios eliciting mild anger or happiness. The result was that test subjects showed signs of anger when they were given an insulting questionnaire and signs of happiness when brought into a room where a stooge was doing funny things. The interesting point is that the reactions of the test group differed from those of the control group, who were not given an anterior adrenaline injection: members of the control group became less emotionally involved with the presented scenarios. Schachter and Singer concluded that autonomic arousal plays a role in emotion insofar as it is perceived as undifferentiated arousal and creates an evaluative need. The subject, feeling her heart beating faster and finding herself in front of an annoying questionnaire, labels her own state as anger and reacts accordingly. Thus Schachter's and Singer's experiment can be seen, as it has been for several decades, as evidence for the claim that emotions cannot be individuated according to a type of bodily arousal they alone involve.

Yet the Schachter/Singer study has been shown to be flawed in several ways. To repeat only one of the arguments brought up against it, it is highly doubtful whether the mere injection of adrenaline causes the same sort of physiological arousal in various subjects in the minutes that follow. The participants might be more sensitive to the happiness- or anger-inducing scenarios because of the adrenaline, but the perception of the anger-eliciting task together with the adrenaline in the blood might cause further bodily changes that are anger-specific, while the adrenaline combined with the funny situation causes bodily arousal that is specific to joy (see Prinz 2004, 70 f.). ${ }^{5}$ So the frequent claim that empirical studies prove that emotion types cannot be distinguished by the bodily reactions they involve is not as well supported by evidence as it might seem at first. ${ }^{6}$ This will become clear in the discussion of recent evidence in the next section. A closer view at the results of recent research render it highly likely that emotions involve something like core-patterns of bodily reactions that are evolutionary acquired and can be found cross-culturally.

5 For further arguments against the Schachter/Singer experiment, see Griffiths 1997 and McNaughton 1989.

6 Walter Cannon (1927) also argues that visceral feedback does not suffice to distinguish various kinds of emotions. However, this objection can be met with the same kind of argument that I have already mentioned, that bodily reactions involve much more than just visceral feedback from the spine and the vagus nerve. 


\section{Bringing in empirical evidence}

The possible sources of evidence of the bodily reactions involved in emotions are numerous and far from having been tested and measured in detail. Even detailed overviews of recent studies are far from issuing clear and stable results. I present a brief and highly selective overview, which aims to show that there is clear evidence against the coincidental-byproduct view and that it is an open question how closely emotion types and discrete physiological responses are related. As has already been mentioned, the place to look for bodily reactions is not only the inner organs and the visceral feedback stemming from them, but also the endocrine system and the somatic nervous system that innervates skeletal muscles, including those of the face.

Indeed, research focused on facial expressions (and more recently on body posture) has been most successful. Paul Ekman has investigated the facial expressions accompanying emotions. By carrying out research on facial expressions for more than thirty years he has established a theory of basic emotions, which are expressed in ways that can be recognized panculturally. Ekman and his colleagues developed the facial action coding system (FACS), which codes observable facial muscle-movements, to test whether their configurations could be identified with the expression of specific emotions across cultures (Ekman 1997). The research on re-identifiable facial expressions in many cultures, including some which barely had any contact with the western world, resulted in the hypothesis that several facial expressions occur and can be recognized universally. The emotions for which Ekman found stable re-occurring patterns are fear, anger, joy, sadness, disgust, and surprise (Ekman 1971; see also Matsumoto et al. 2010 for a current synopsis).

Apart from the visual observation of facial expressions, which Ekman and colleagues performed, nowadays there is a growing amount of research using facial electromyography. Facial EMG directly measures muscle activity by detecting and amplifying the electrical impulses generated by muscle fibers when they contract. The lowering and contraction of the brows, for example, has been observed as part of the expressive reaction to unpleasant stimuli. It is involved in producing frowns. Therefore, the firing of motor units in this muscle region can be expected if a stimulus is judged to be unpleasant. But facial EMG studies have also found that activity of the corrugator muscle, which lowers and contracts the eyebrow, varies inversely with the valence of the observed stimuli and the reported emotional state, which means that the muscle, in neutral situations, is under slight tension and relaxes in the presence of pleasant stimuli (Schwartz et 
al. 1979). ${ }^{7}$ The corrugator muscle can be described as a permanent bodily valence marker that sends proprioceptive feedback to the brain, which indicates the presence of pleasant, unpleasant, and neutral stimuli.

While the research on facial expressions alone only led to the discovery of the six aforementioned basic emotions (or rather seven, since Ekman later added contempt to the list), recent studies have extended the view to combinations of facial expression and body posture and found evidence for cross-culturally stable patterns of pride and shame as well (Tracy and Robbins 2007a; 2007b and Clark 2010; for discussion, see below). It is likely that further research will reveal evidence for even more emotion types. Facial expressions do not occur in isolation but have been shown to be systematically associated with physiological responses, such as skin conductance, cardiovascular activation, and somatic activity (Mauss et al. 2005), and with neuroendocrine activity (Lerner et al. 2005). Lerner et al. were able to show that fear expressions are associated with elevated heartbeat and cortisol levels, while anger and disgust show reduced levels.

With regard to the reactions of the autonomic nervous system, the evidence is ambiguous. Yet hardly anybody would claim today that there is no covariance between reactions of the nervous system and particular features of emotions at all. Even Lisa Feldman-Barrett (2006) who assumes that there are no emotion kinds with a unique and invariant autonomic signature, suggests that patterns of physiological responses can be divided into more general dimensions of threat and challenge, and positive and negative valence (see also Taylor 1991; Cacioppo et al. 2000). If we look at emotion patterns rather than individual variables of autonomic reactions, no current position would deny differences among coarsegrained emotional dimensions (Mauss and Robinson 2009; Harrison et al. 2013).

Recent overviews even suggest that emotion types can be much better individuated via patterns of autonomic nervous system (ANS) arousal than was previously thought. A main reason for this is its greater sensitivity to fine-grained differences and to the various sources of those differences. While most studies are, to a large degree, based on measures of cardiovascular, respiratory, and skin conductance responses, when it comes to individuation a lot depends on measuring more and more correct factors to individuate a certain emotion type. Sylvia Kreibig (2010) points out that, in the case of disgust, measurements of the increased heart rate and skin conductance tend to resemble other negative emotions like fear and anger. Surprisingly, there are few tests that look at gastrointestinal responses that might correspond to disgust, although lay association

7 See also Laird 2007 and Matsumoto et al. 2010 for general overviews of the feedback generated by facial expressions. 
could draw such a connection and the few studies that tested for stomach electrical activity could actually find reactions.

Fear is among those emotions for which there are stable results for a unique ANS pattern already. Fear studies have used a number of different fear-induction paradigms and identified a broad pattern of sympathetic activation that includes increased heart rate, narrowing of the blood vessels and electrodermal activity, accompanied by an increase in respiratory activities associated with a decrease in blood carbon dioxide levels (Kreibig 2010; Harrison et al. 2013). Anger (elicited through harassment or personalized recall) is associated with a general increase in sympathetic activity as well; it also includes an increase in heart rate and systolic and diastolic blood pressure, but, unlike fear, anger is associated with total peripheral resistance (Harrison et al. 2013). Some studies also found that in anger there is an increase in forehead temperature, probably due to a general increase in facial circulation (Stemmler et al. 2001). Analogously, an increase in facial blood flow in anger was found in rhesus monkeys (Nakayama et al. 2005). Happiness, like many negative emotions, is associated with cardiac activation, increased blood pressure, and increased respiratory activity. However, in contrast to many negative emotions, it is associated with peripheral vasodilation (Harrison et al. 2013). When comparing different types of emotions there are similarities such that most positive and negative emotions involve some kind of arousal, which tends to be stronger in negative emotions such as fear and anger. However, taking a closer look at fear and anger in particular, it turns out that anger appears to be more strongly associated with vascular activity than fear, but less strongly associated with cardiac activity. These results render it highly likely that emotions involve something like core-patterns of bodily reactions that are evolutionary acquired and can be found cross-culturally (see also Colombetti 2014).

It must be noted, however, that there is a huge amount of data lacking in this field and a considerable amount of confusion and seemingly contradictory results brought forth in recent decades. For example, while Cacioppo et al. (2000) found fear to be associated with a greater heart rate increase than anger, Labouvie-Vief et al. (2003) found that imagery-induced fear and anger elicited comparable increases in heart rate. A plausible explanation for such differences is that many studies neglect the importance of context-dependent aspects of emotional reactions. Different studies compared, for example, the physiology of fear in situations as different as hearing a loud noise, looking at a picture of an amputated leg, giving a public speech, or imagining an intruder in one's house (Bradley 2007). Cardiac reactions also tend to differ when subjects process affectively similar events in different types of tasks. The results of the study by Labouvie-Vief et al., for example, might be explained by the fact that heart 
rate tends to decelerate during unpleasant picture-viewing, while it accelerates when unpleasant mental imagery is evoked (Lang et al. 1990). The context of perception or imagination has a strong influence on the heart rate. A more obvious case is that the psychophysiology of emotion in action can differ from vigilant anticipation. Even without further evidence, one can make good guess about the difference in heart rate measured when somebody meets a snake while she is jogging through the woods compared to somebody lying on the couch and seeing a snake suddenly appearing on TV. Therefore, the parameters of each task, which have implications for the motivational action, have to be carefully distinguished before comparing studies. While one could try to come up with bodily arousal patterns relative to the situations in which they occur, finding stable patterns is further complicated by the degree to which individual differences influence patterns of physiological reactions, including interpersonal differences in gender, age, temperament, and intellectual capacity, such as imagination and memory, just to name a few (Feldman-Barrett, Russell 2015).

Another complication stems from the fact that the presence of specific emotions is not only inferred on the basis of physiological reactions, but also on the basis of the behavioral actions they cause, and these behaviors tend to vary depending on contextual support. In response to an electric shock, even rats show a variety of different behaviors depending on their actual environment. If there is an exit, they escape; if there is another rat, they attack; and if there is neither, they freeze (Lang 1990). This tactical nature of emotional behavior, which is already present in animals far less intelligent than humans, constrains the efforts of psychophysiologists to make general inferences regarding the physiology of specific emotional states since freeze, fight, and flight reactions pose different metabolic demands and therefore also influence the emotional arousal evoked by emotional processes (Harrison et al. 2013).

The evidence above makes the Jamesian hypothesis, that a specific emotion has a specific physiological pattern, look too general and difficult to test since the physiological pattern of an emotion depends on the individual, on its concrete circumstances, and on the kind of task in which the emotion-eliciting cue appears. This not only calls for clean parameters to be defined in studies on the psychophysiology of emotions. It also raises the question of whether it makes sense to think of the bodily arousal involved in emotions in terms of essential ingredients, i.e., in terms of a pattern with stable features that can be reidentified in the various occurrences of an emotion-type in a subject over time as well as among different subjects. 


\title{
4 The functionalist objection
}

We have seen that there is considerable evidence for re-occurring patterns of bodily reactions in emotions, but we have seen as well that the evidence does not suffice to identify sets of essential elements for each emotion as the essential-ingredient view would demand. The essential-ingredient view has no answer to a simple functionalist argument such as the one Nussbaum has constructed to support her view:

\begin{abstract}
All human experiences are embodied, and thus realized in some kind of material process. In that sense, human emotions are all bodily processes. But the question is, are there any bodily states or processes that are constantly correlated with our experiences of emotion, in such a way that we will want to put that particular bodily state into the definition of a given emotion-type? And here we run up against an issue well-known to biological researchers: the plasticity of the human organism, or, in other words, the multiple realizability of mental states (Nussbaum 2001, 58f.).
\end{abstract}

Nussbaum here refers to the functionalist paradigm in philosophy of mind that was elaborated by Hilary Putnam (1960) and others in the context of computationalist theories of mind. At the heart of functionalism lies the idea that mental states like ours might be realized in several ways and should not be type-identified with reference to their particular neural underpinnings but, instead, with reference to their functional role.

Multiple realizability is a central concept in this context. It can be understood in two ways: first, with regard to an individual and, second, with regard to different kinds of living organisms. With regard to the individual, the findings on the plasticity of the brain suggest that the brain is a functional organ where, for example, after a lesion the functions realized by the damaged areas can come to be realized by other areas of the brain. Therefore, it does not make sense to claim that a particular arrangement of neurons in a particular individual realizes one particular type of mental state. Across species there are obviously functionally identical mental states, like pain or hunger, in most mammals, while at the same time we would not expect the neural machinery realizing a pain-state in a rat to be precisely the same as in humans. Even with regard to individuals from the same culture Nussbaum argues, "if we said that grief is always of necessity accompanied by the firing of so-and-so many neurons of such and such type, we would be likely to find hundreds of cases for which this just isn't quite right" (Nussbaum 2001, 59). Nussbaum takes the central nervous system to be part of the bodily realization of an emotion, which in a broader sense is, of course, correct. But the Jamesian hypothesis in particular takes emotions to be percep- 
tions of arousal in the peripheral nervous system. So the question is whether we find distinct patterns of bodily arousal there that can be perceived by certain parts of the brain (James thought these to be subcortical sensorimotor areas), and not whether the neurons in the brain form distinct patterns that can be identified. ${ }^{8}$

But Nussbaum's argument can be turned into an argument against James: it is not only the brain that is plastic, but the whole nervous system. Therefore one can have doubts about the possibilities of finding exactly the same reactions, e.g., in the endocrine system, in the same person over several years. And one can also have doubts about whether we find the same reactions in the endocrine system when observing fear in a female baboon compared with an old man. Put this way, it becomes easy to see what position Nussbaum is denying: she does not want to commit herself to the claim that there is a single pattern of neuronal activity or bodily arousal that is activated whenever somebody experiences a certain type of emotion. Instead, the functionalist idea Nussbaum refers to claims that a fear-state can be realized in various ways, and that emotions should therefore be type-identified with regard to the mental evaluations they involve.

Yet what remains unclear is what explanatory role is left for the body in such a functionalist account. On the one hand, Nussbaum claims, "all human experiences are embodied, and thus realized in some kind of material process. In that sense, human emotions are all bodily processes" (Nussbaum 2001, 58). So Nussbaum seems to commit herself to some version of materialism, as most functionalists do. But on the other hand, Nussbaum takes it to be a virtue of her definition that it includes bodiless beings such as gods and angels.

Whether we believe that bodiless substances exist or not, the reason it makes sense to imagine a bodiless substance having genuine emotions is that it makes sense to imagine that a thinking being, whether realized in matter or not, could care deeply about something in the world, and have thoughts and intentions associated with such attachments. And that's all we really require for emotion (Nussbaum 2001, 60).

We have already seen that not even James would deny the mere conceptual possibility of such bodiless emotional creatures. Yet for Nussbaum, the reference to them is meant to support her view that emotions should be type-identified with regard to the evaluation that emotions involve.

8 I have already mentioned that the view I want to defend here departs from the Jamesian hypothesis. But with respect to this particular point, the embodied-emotions hypothesis I am discussing here, just like the Jamesian hypothesis, does not depend on the question of whether there is a particular brain state that realizes an emotion, but rather on whether there is a particular set of bodily reactions that does so. However, Nussbaum denies both claims. 
One may find it confusing, though, that Nussbaum refers to the functionalist argument of multiple realizability, but at the same time claims that thinking beings do not need to be realized in matter at all. Most functionalist theories that argue for multiple realizability do not deny the important causal role of matter in the making of mental states. They simply claim that several different material arrangements can fulfill the same causal roles. Multiple realizability is not equivalent to the complete arbitrariness of the realizing arrangement of matter. Yet what Nussbaum seems to have in mind is a position that claims that emotions are mental evaluations that can be realized in all kinds of ways. Coincidentally, in all animals, including humans, emotions are realized in their brains and bodies. But the ways in which they are realized vary enormously so that it does not make sense to refer to them when looking for features to classify emotions.

This appears to be a radical version of what Lawrence Shapiro (2004) calls the "separability thesis." According to the separability thesis, it is perfectly possible for a humanlike mind to exist in a non-humanlike body. Shapiro rejects this view, arguing that e.g. visual perception in humans depends fundamentally on the way we move our heads to gain information about the distance of objects. Shapiro makes similar points with regard to the other sensory modalities and concludes that "a description of various perceptual capacities cannot maintain body-neutrality and ...an organism with a nonhuman body will have non-visual and auditory psychologies" (Shapiro 2004, 190). What Shapiro means by bodyneutrality is precisely what Nussbaum is arguing for: the view that the mind is a program that can be realized in several kinds of bodies and can be characterized in abstraction from the body. Like Shapiro, I think that the ways in which the body contributes to our special ways of perceiving the world have to be taken into account. There are a variety of ways in which this idea can be explained, by giving more or less weight to the precise role of the autonomic nervous system, several brain structures, and cognitive processes. But for any kind of naturalist theory, functionalist or not, Nussbaum's coincidental-byproduct view is a slightly-too-arbitrary description of the role of bodily reactions in emotions.

Furthermore, the evidence discussed so far clearly shows that the bodily reactions are far from arbitrary. Consider Nussbaum's example of rage again. Nussbaum reports that while many men say that they associate rage with a boiling feeling, she associates rage with a tense feeling around the neck and sometimes a headache occurring the next day. With regard to the studies discussed above, there might be several reasons for these differences: gender reasons might be important, but there is also evidence that people have different abilities when it comes to sensing their own heart rate (Barrett et al. 2004) and sensing their own respiratory frequency. Parts of this interoceptive awareness might also improve through training (Daubenmier et al. 2013; Bornemann et al. 2015). Yet 
tensed muscles, increased heart rate, and elevated body temperature all fit into the prototypical pattern of bodily arousal that is generally associated with rage. What would be disturbing is if Nussbaum reported feeling her heart beat slow down whenever she is angry, or if she associated it with a relaxation of her muscles from the corrugator all the way down to the belly and the toes. My guess is that in such a case we would doubt whether the feeling that Nussbaum describes could be labeled as a case of anger at all. This intuition, together with the evidence discussed above, should suffice to disqualify an approach that denies any significant functional role of the bodily changes involved in emotions altogether.

Still, there is a lesson to be learned from Nussbaum: if James's claim, that there are specific patterns of bodily arousal in humans that can be used to type-identify emotions, is taken literally, it seems hard to reconcile with the functionalist idea of multiple realizability. Yet the idea that all fear-states in all mammals under all circumstances should involve exactly the same pattern of arousal sounds unlikely. We need a better definition of what these patterns are and what it means to re-identify them in various organisms in various situations. The Jamesian question needs to be situated in a broader theoretical framework in order to be answered.

\section{Bringing in Dewey}

The essential-ingredient view and the coincidental byproduct view both seem to be inadequate with regard to current evidence. Bodily arousal plays an important role in emotions and there appear to be stable and evolutionarily acquired patterns at least for some coarse-grained emotion categories. But emotional episodes can differ strongly and are sensitive to many contextual factors. It is therefore unlikely that we will ever find crossculturally stable essential ingredients for each emotion type.

An essential feature of emotions is their motivational force. Emotions have been described as action-tendencies that respond to urgent situations in the environment (Frijda 1986). When it comes to explain this urgency bodily arousal obviously plays a constitutive role. We feel motivated to approach somebody in an aggressive way because of the increased heart rate, muscle tension, blood pressure, and so on. If emotions are described and individuated as action-tendencies the bodily arousal appears to be relevant and specific but not entirely stable over all possible contexts. Emotional episodes can differ strongly depending on the context in which they take place. Furthermore the bodily reactions involved in emotions are not hardwired but rather skillful. From 
birth on we adopt and train certain respiratory cycles, ways to tense and relax our muscles and show certain facial expressions and we also learn in which situations these emotional expressions are seen as appropriate reactions in the culture we live in. Therefore it is not only single emotional episodes that might differ, different histories of coping with different social environments might lead to slightly different patterns of bodily arousal in individuals and among cultures.

Such an action-oriented view has been articulated for perception and emotion by John Dewey (1895; 1896). In his writing on emotion Dewey aims to unite Darwin's and James's approaches to emotions. He criticizes James for his focus on emotional feelings as being constituted by bodily arousal. Instead he highlights that emotions are habituated actions and discusses this using the famous example of fear of a bear: "It is not the idea of the bear, or the bear as object, but a certain act of seeing, which by habit, whether inherited or acquired sets up other acts.” (Dewey 1895, 17). Dewey here also claims that the emotional episode and the emotional object are inseparable and that the emotional object is constituted by the emotional episode. I disagree with this view and will leave it aside for now. The way Dewey puts the focus on action becomes clear when looking at his critique of the reflex arc concept in psychology and the way he takes sensation and motor activity to be closely intertwined and to likewise constitute perception. The act of looking, executed by the whole organism, is what is primary and both sensation (i.e., the processing of the input) and movement are parts of this act, which constitutes perception. Action and sensation are closely intertwined; they "have been so often bound together to reinforce each other, to help each other out, that each may be considered practically a subordinate member of a bigger coordination" (Dewey 1896, 359). While the hand permanently depends on the control and stimulation of the visual information, vision in turn must be stimulated and controlled by the body's movements. If the eye is not kept fixed on the goal of grasping, if there is no proprioceptive feedback about the body's posture, the task cannot be fulfilled.

Arguments for such an action-oriented approach with regard to emotions can be grounded on empirical data from various sources. From a psychophysiological point of view it can be doubted whether the input- and output-side of an emotion can be clearly separated. Several studies suggest that emotional behavior (e.g., bodily postures, facial and vocal expressions), which is commonly regarded as a mere output, can modulate emotions and sometimes even trigger them (see Niedenthal et al. 2005 for an overview). To test the influence of facial feedback on emotions and emotional feelings, for example, participants were given non-emotional tasks to guide the production of facial expressions without cueing the emotional meaning of the expression. Participants then had to evaluate certain stimuli, like cartoons, with regard to whether they were funny. Find- 
ings suggest that the intensity and quality of the participants' manipulated facial expression affected the intensity of their self-reported emotional feelings as well as their autonomic responses. Facial expressions modulate emotions and sometimes even trigger them (Laird 2007). When facial feedback is reduced through Botox injections, finer notes of emotional feelings tend to be reduced (Davis, Senghas, Brandt Ochsner 2010, chapter 2). Bodily postures seem to impact emotional experience in a similar way. In a study conducted by Stepper and Strack (1993), participants' bodily posture was manipulated by asking them to adopt one of two conventional working positions, an upright or a slumped posture. Participants then received positive feedback concerning their performance on an achievement task. Those who received success-feedback in the slumped posture felt less proud and reported being in a worse mood than participants in the upright position (for similar results see Duclos et al. 1989). A different study found that an emotion-specific tone of voice has been found to amplify emotional experience as well (Hatfield et al. 1995).

Taken together, expressive behaviors facilitate, modulate, and produce corresponding emotional reactions and feelings. This suggests that in emotional processing input and output processing pathways are not clearly separated. Rather, emotional processing works in feedback loops: the organism produces output that affects its input in a systematic way. It not only receives stimuli from the world but also is a source of stimulation to itself. Getting angry in a certain situation might not depend on the input received from the external world alone. The organism's entire situation including bodily posture and current facial expression plays a role as well.

The data from psychophysiology and neuroscience discussed so far suggest that emotions cannot be adequately described by the essential-ingredient-view that bodily ingredients constitute emotional feelings. What unifies a certain emotion type, according to an action-oriented-view is not its particular bodily profile alone but rather the bodily profile seen as constituting a certain action-tendency. We find evidence for bodily responses constituting broad and stable emotion categories like fear as a danger warning system that prepares for flight and anger as a response to an offense that prepares for aggressive behavior or guilt as a response to a rule violation that prepares to make amends. But the bodily profile of emotional episodes can vary strongly with regard to the particular context. Increase of heart rate differs when meeting a bear while already running to meeting a bear when sitting relaxed in a chair. Such live situations differ largely from seeing a bear on television. Furthermore we acquire different embodied skills throughout our lives and through our interactions with different social surroundings. When Aristotle describes anger as being accompanied by the boiling of the blood around the heart and Nussbaum describes anger as being accompanied by 
a slight tension at the back of the neck, they point to different embodied skills that both contribute to largely the same action tendency, namely aggressive behavior. Yet there are different ways of showing aggressive behavior. Some are more direct, some more subtle, some impulsive, some planned. These differences in how we are motivated to show aggression in anger is shaped by a learning process that takes place in a social setting. But these learning processes do not start with the body as a blank slate. Anger involves an increase of heart rate, blood pressure and muscle tension. So while the present approach can integrate variations in emotional action tendencies, this does not make the bodily constituents of these action-tendencies arbitrary. If Aristotle would describe his being angry as being accompanied by a pleasant relaxation of the muscles or Nussbaum would say that anger for her really feels as if the blood circulation would slow down in a way that puts her in a peaceful state of mind, I guess that we would rather doubt that they are describing that particular emotion that we call anger.

\section{Conclusion and open questions}

I have introduced the essential-ingredient view that is based on a certain reading of William James. I further discussed the objections that cognitivists such as Martha Nussbaum have raised against James and the coincidental-byproduct view that these authors defend. I argued that with regard to current empirical evidence from psychophysiology the coincidental-byproduct view is untenable. I also argued that James's view understood as an essential-ingredient view is untenable and needs to be put into a larger framework. Dewey already offers a rereading of James focusing on the action tendencies that are constituted by the bodily arousal rather than on the feeling. In this vein I suggest to think of emotions as being action-oriented and think of the bodily arousal as the functional equipment that realizes certain action-tendencies in various contexts in various ways. A dimension of emotions that I completely left out of the present paper is the cognitive or normative dimension of emotions. ${ }^{9}$ Emotions are not just bodily responses they have intentional objects and can be described as being subject to semantic and social norms. A current embodied action-oriented approach should account for the way emotions represent the world and for the complex

9 But see Hufendiek (2016). 
ways in which emotions relate to other emotions, perceptions and thoughts in a naturalist framework.

\section{References}

Adelmann, P. \& Zajonc, R. (1989). “Facial Efference and the Experience of Emotion.” In: Annual Review of Psychology, vol. 40, 249-280.

Barrett, L. F. et al. (2004). "Interoceptive Sensitivity and Self-Reports of Emotional Experience." In: Journal of Personality and Social Psychology, vol. 87, no. 5, 684-697.

Barrett, L. F. et al. (2006): “Are Emotions Natural Kinds?” In: Perspectives on Psychological Science 1, 28-58.

Bornemann, B. et al. (2013). "Differential Changes in Self-Reported Aspects of Interoceptive Awareness through 3 Months of Contemplative Training." In: Frontiers in Psychology, vol. 5, 1504.

Bradley, M. (2000/2007). “Emotion and Motivation.” In: J. Cacioppo, G. Tassinary \& G. Bernston (Eds.). Handbook of Psychophysiology. Cambridge: Cambridge University Press. 602-642.

Cacioppo, J., Priester, J. \& Bernston, G. (1993). "Rudimentary Determinants of Attitudes. II: Arm Flexion and Extension have Differential Effects on Attitudes." In: Journal of Personality and Social Psychology, vol. 65, no. 1, 5-17.

Cacioppo, J., Tassinary, G. \& Bernston, G. (2000). Handbook of Psychophysiology. Cambridge: Cambridge University Press.

Cannon, W. (1927). "The James-Lange Theory of Emotion: A Critical Examination and an Alternative Theory.” In: American Journal of Philosophy, vol. 39, 106-124.

Cannon, W. (1936). Bodily Changes in Pain, Hunger, Fear, and Rage. New York: Appleton-Century.

Cannon, W. et al. (1988). "Autonomic Arousal Feedback and Emotional Experience: Evidence From the Spinal Cored Injured." In: Journal of Personality and Social Psychology, vol. 54, no. 5, 820-828.

Damasio, A. (1994). Descartes' Error. Emotion, Reason and the Human Brain. London: Vintage Books.

Damasio, A. (1999). The Feeling of What Happens: Body and Emotion in the Making of Consciousness. New York: The Ecco Press.

Damasio, A. (2010): Self Comes To Mind: Constructing the Conscious Brain. New York: Pantheon Books.

Darwin, C.: (1872/2009). The Expression of The Emotions in Man and Animals. London: Penguin Books.

Daubenmier et al. (2013). "Follow Your Breath: Respiratory Interoceptive Accuracy in Experienced Meditators.” In: Psychophysiology, vol 50, no. 8, 777-789.

Davis, J. et al. (2010). "The Effects of BOTOX-Injections on Emotional Experience." In: Emotion, vol. 10, no. 3, 433-440.

Dewey, J. (1895). "The Theory of Emotion 2. The Significance of Emotions.” In: Psychological Review, vol. 2., $13-32$.

Dewey, J. (1896). “The Reflex Arc Concept in Psychology.” In: The Psychological Review, vol. 3, no. 4, 357-370. 
Ekman, P. (1971). “Universals and Cultural Differences in Facial Expressions of Emotion.” In: J. Cole (Ed.). Nebraska Symposium on Motivation 4. Lincoln: University Press of Nebraska, 207-282.

Ekman, P. (2003). Emotions Revealed. Understanding Faces and Feelings. London: Orion Books.

Ekman, P. \& Rosenberg, E. (1997). What the Face Reveals. Basic and Applied Studies of Spontaneous Expressions Using the Facial Action Coding System. New York: Oxford University Press.

Ekman, P., Levenson, W. \& Friesen, W. (1983). “Autonomic Nervous System Activity Distinguishes among Emotions.” In: Science, vol. 221, no. 4616, 1208-1210.

Ellsworth, P. (2014). "Basic Emotions and the Rocks of New Hampshire." In: Emotion Review, vol. 6, 21-26.

Feldman-Barrett, L. (2006). “Are Emotions Natural Kinds?” In: Perspectives on Psychological Science 1, 28-58.

Feldman-Barrett, L. \& Russell, J. (2015). “Ten Misconceptions About the Psychological Construction of Emotion.” In: L. Feldman-Barrett \& J. Russell (Eds.): The Psychological Construction of Emotion. New York: Gilbert, 45-79.

Frijda, N. (1986). The Emotions. Cambridge: Cambridge University Press.

Griffiths, P. (1997). What Emotions Really Are. The Problem of Psychological Categories. Chicago: University of Chicago Press.

Harrison, N., Kreibig, S. \& Critchley, H. (2013). “A Two-Way Road: Efferent and Afferent Pathways of Autonomic Activity in Emotion.” In: J. Armony \& P. Vuilleumier (Eds.). The Cambridge Handbook of Human Affective Neuroscience. Cambridge: Cambridge University Press, 82-106.

Hufendiek, R. (2016). Embodied Emotions. A Naturalist Account to a Normative Phenomenon. New York: Routledge.

Hurley, S. (1998). Consciousness in Action. Cambridge, MA: Harvard University Press.

Hurley, S. (2001). "Perception and Action. Alternative Views." In: Synthese, vol. 129, no. 1. $3-40$.

Kreibig, S. (2010). “Autonomic Nervous System Activity in Emotion: A Review.” In: Biological Psychology, vol. 84, no. 3, 394-421.

Labouvie-Vief et al. (2003). "Age and Gender Differences in Cardiac Reactivity and Subjective Emotion Response to Emotional Autobiographical Memories.” In: Emotion, vol. 3, no. 1, $115-126$.

Laird, J. (2007). Feelings. The Perception of Self. Oxford: Oxford University Press.

Laird, J. (1974). "Self-Attribution of Emotion: The Effects of Expressive Behavior on The Quality of Emotional Experience." In: Journal of Personality and Social Psychology, vol. 29 , no. 4, $475-486$.

Laird. J. \& Lacasse, K. (2014). "Bodily Influences on Emotional Feelings: Accumulating Evidence and Extensions of William James's Theory of Emotions." In: Emotional Review, vol. 6, 24-37.

Lang, P., Bradley, M. \& Cuthbert, B. (1990). “Emotion, Attention and the Startle Reflex." In: Psychological Review, vol. 97, no. 3, 377-395.

Lang, P., Bradley, M. \& Cuthbert, B. (1998). "Emotion, Motivation, and Anxiety: Brain Mechanisms and Psychophysiology." In: Society of Biological Psychiatry, vol. 44, no. 12, $1248-1263$. 
Lazarus, R. (1991). Emotion and Adaptation. New York: Oxford University Press.

Lerner et al. (2005). "Facial Expressions of Emotions Reveal Neuroendocrine and

Cardiovascular Stress Responses.” In: Biological Psychiatry, vol. 58, 743-750.

Mauss, B. et al. (2005). "The Tie That Binds? Coherence Among Emotion Experience, Behavior and Physiology." In: Emotion, vol. 5, no. 2, 175-190.

Mauss, B. \& Robinson, M. (2009). "Measures of Emotion: A Review." In: Cognition and Emotion, vol. 23, no. 2, 209-237.

Niedenthal, P. et al. (2005). "Embodiment in the Acquisition and Use of Emotion Knowledge." In: L. Feldman Barrett, P. Niedenthal \& P. Winkielman (Eds.). Emotion and Consciousness. New York: Guilford Press, 21-51.

O’Regan, K. \& Noë, A. (2001). “A Sensorimotor Account of Vision and Visual Consciousness." In: Behavioral and Brain Sciences, vol. 24, no. 5, 939-1031.

Ratcliff, M. (2005). "William James on Emotions and Intentionality." In: International Journal of Philosophical Studies, vol 13, no. 2, 179-202.

Reisenzein, R. \& Stephan, A. (2014). "More on James and the Physical Basis of Emotions.” In: Emotion Review, no. 6, 35-46.

Schwartz, G., Ahern, G. \& Brown, S. (1979). "Lateralized Facial Muscle Response to Positive and Negative Emotional Stimuli.” In: Psychophysiology, vol. 16, 561-571.

Shapiro, L. (2004). The Mind Incarnate. Cambridge, MA: MIT Press.

Shapiro, L. (2010). Embodied Cognition. New York: Routledge.

Solomon, R. (1973/2003). “Emotions and Choice.” In: R. Solomon. Not Passion's Slave. Emotions and Choice. Oxford: Oxford University Press. 3-24.

Stepper, S. \& Strack, F. (1993). "Proprioceptive Determinants of Emotional and Nonemotional Feelings." In: Journal of Personality and Social Psychology, vol. 64, no. 2, 211-220.

Strack, F., Martin, L. \& Stepper, S. (1988). "Inhibiting and Facilitating Conditions of the Human Smile: A Nonobtrusive Test of the Facial Feedback Hypothesis.” In: Journal of Personality and Social Psychology, vol. 54, no. 5, 768-777. 